\title{
LA FIGURA DEL FACILITADOR EN CASOS DE ABUSO SEXUAL A PERSONAS CON DISCAPACIDAD INTELECTUAL
}

\section{The figure of the helper advisor in cases of sexual abuse against people with intellectual disabilities}

\author{
Almudena Martorell Cafranga \\ Fundación Carmen Pardo-Valcarce \\ Alberto Alemany Carrasco \\ Fundación Carmen Pardo-Valcarce. Unidad de Atención a Victimas con Discapacidad Intelectual. \\ C/ Monasterio de las Huelgas, 15. 28049 Madrid \\ alberto.alemany@fcpv.es
}

Recepción: 30 de junio de 2016

Fecha de aceptación definitiva: 31 de enero de 2017

RESUMEN: En el presente trabajo analizamos las principales barreras que se encuentran las personas con discapacidad intelectual que han sufrido un abuso sexual cuando acceden al sistema de justicia. Frente a estas barreras, la Unidad de Atención a Víctimas con Discapacidad Intelectual de la Fundación Carmen Pardo-Valcarce propone la inserción en el proceso judicial de la figura del facilitador. La entrada en vigor en 2015 de la Ley 4/2015, de 27 de abril, del Estatuto de la Víctima del Delito supone una oportunidad ejemplar para garantizar la incorporación de las propuestas de apoyo que implica la inserción de la figura del facilitador en los procesos judiciales en los casos en los que la víctima es una persona con discapacidad intelectual. Analizamos también el impacto que la figura del facilitador ha tenido en el paso por la justicia de las personas con discapacidad intelectual víctimas de abuso sexual, haciendo especial hincapié en los casos que han sido sobreseídos en fase de instrucción.

Palabras Clave: víctima; discapacidad intelectual; vulnerabilidad; abuso sexual; facilitador; barreras; justicia; apoyo. 


\section{LA FIGURA DEL FACILITADOR EN CASOS DE ABUSO SEXUAL A PERSONAS CON DISCAPACIDAD INTELECTUAL \\ ALMUDENA MARTORELL CAFRANGA Y ALBERTO ALEMANY CARRASCO}

AвSTRACT: In this paper we analyse the main barriers that people with intellectual disabilities who have suffered sexual abuse have to face when they access the Justice system. Regarding these barriers, the Victim Support Unit for People with Intellectual Disabilities of the Fundación Carmen Pardo-Valcarce proposes the inclusion in the judicial process of a helper advisor. The entry into force in 2015 of the Law 4/2015, in 27 April, the Statute of the crime victim represents an exemplary opportunity to ensure the incorporation of support proposals involving the insertion of the helper advisor in the judicial process in cases where the victim is a person with intellectual disabilities. In this paper we analyse the impact of the helper advisor, with particular emphasis on cases that have been dismissed under instruction.

KEY WORDS: victim; intellectual disability; vulnerability; sexual abuse; helper advisor; barriers; justice; support.

\section{Abuso y discapacidad intelectual}

OMO HAN INDICADO DIFERENTES ESTUDIOS en nuestro país (Verdugo y Bermejo; 1995 y Verdugo, Alcedo, Bermejo y Aguado, 2002), las personas con discapacidad intelectual presentan una elevada vulnerabilidad al abuso sexual (Horner-Jonhson y Drum, 2006), con estudios que alertan de que hasta un $80 \%$ podrá ser víctima de ello a lo largo de su vida (Brown, Stein y Turk, 1995; Horner-Johnson y Drum, 2006; McCarthy y Thompson, 1997; Sobsey, 1994; Verdugo, Alcedo, Bermejo y Aguado, 2002). Además, las consecuencias traumáticas de estas situaciones de abuso se ven acrecentadas por la carencia de sistemas de prevención e intervención a nivel social (Johnson, 2012).

Han sido identificados los factores individuales que explican esta realidad, pero parece claro concluir que lo que se esconde detrás es la falta de empoderamiento, de autodeterminación y, por tanto, de igualdad de derechos de este grupo de personas. O, como apunta Huete (Huete, 2012), un problema derivado de tratarse la discapacidad intelectual de una condición que conduce casi inexorablemente a la exclusión social. Cabe recordar, además, que la exclusión social se configura y retroalimenta desde los elementos no sólo sociales, sino también económicos y jurídicos incidiendo así éstos, igualmente, en el fenómeno que ocupa el presente trabajo (Anaut-Bravo, Arza y Álvarez, 2014).

Esta reflexión es aplicable a otros colectivos históricamente excluidos, como la mujer hasta hace un siglo y la gran incidencia de abuso sexual y explotación, o las personas de otras razas. Por ello se trata de un fenómeno macrosistémico, que no podrá ser abordado en su totalidad sin un cambio del modelo con el que la sociedad entiende y percibe la discapacidad intelectual.

Desde la Fundación Carmen Pardo-Valcarce, aunque se trabaja diariamente para propiciar ese cambio macro, siendo conscientes de la cantidad de abusos, principalmente sexuales, entre los alumnos y trabajadores con discapacidad intelectual, se puso en marcha en 2010 la Unidad de Atención a Víctimas con Discapacidad Intelectual, UAVDI. Se comenzó con el foco en el abuso sexual, pero como se desprende de lo 
que se ha expuesto al comienzo, el problema no reside sólo en la vulnerabilidad a este tipo concreto de abuso. Por lo que, al ofrecer el acompañamiento terapéutico y policial y judicial, han empezado a identificarse otras problemáticas escondidas y que explican de nuevo que se trata una realidad que radica en la exclusión: prostitución forzosa, trata con fines de explotación sexual, explotación laboral, redes de mendicidad, acoso laboral, bullying y delitos de odio o abuso financiero entre otros. Realidades complejas que quizá se expliquen mejor desde la formulación de la pregunta de por qué es una población a la que se le puede privar del voto, o donde la esterilización forzosa está a la orden del día o que incluso se considera por muchos que no deben tener descendencia por entenderse que en ellos mismos reside la incapacidad de cuidar de la misma.

\section{Ejes de intervención en la UAVDI de la Fundación Carmen Pardo-Valcarce}

Ya más concretamente, la UAVDI de la Fundación Carmen Pardo-Valcarce trabaja por abordar el problema desde tres ejes fundamentales de actuación: la investigación, la prevención y sensibilización, y la intervención.

El eje de investigación pretende dar respuesta a un doble objetivo: desarrollar herramientas e instrumentos que garanticen que el acceso a la justicia de la persona con discapacidad intelectual sea en condiciones de igualdad y desarrollar herramientas e instrumentos que permitan a los profesionales clínicos evaluar y trabajar sobre las secuelas del abuso y el maltrato en víctimas con discapacidad intelectual.

Desde el eje de prevención y sensibilización se persiguen dos objetivos principales: reducir la vulnerabilidad de las personas con discapacidad intelectual frente al abuso y el maltrato y potenciar canales de detección de situaciones de abuso y maltrato a personas con discapacidad intelectual.

Por último, el eje de intervención se articula con un doble objetivo: garantizar que el acceso a la justicia de la persona con discapacidad intelectual sea en condiciones de igualdad y con el menor coste emocional posible, a través de la inserción en los procesos de la figura del facilitador -cuya labor se desarrolla en este artículo- y garantizar que las consecuencias psicosociales negativas derivadas de la situación de victimización criminal sean paliadas a través del acompañamiento psicoterapéutico. La UAVDI, por tanto, atiende de manera gratuita, en todo el territorio nacional, a víctimas con discapacidad intelectual de cualquier forma de abuso o maltrato (abuso y agresión sexual ${ }^{1}$, violencia física, abuso financiero, violencia de género, bullying o ciberacoso, entre otros).

De esta manera, desde la puesta en marcha de la UAVDI en 2010 se ha dado servicio a un total de 430 casos. Cabe destacar el aumento significativo que tiene el número de derivaciones año tras año (ver Gráfico 1). Este hecho se explica, en parte, a través del mayor conocimiento de los servicios de la UAVDI en todo el territorio nacional y gracias a la mejoría en los procesos detección, relacionada con la formación impartida

1 Las menciones al concepto de "abuso sexual” aglutinan los principales delitos contra la libertad e indemnidad sexuales (abuso y agresión sexual). 
por la unidad (más de 2.000 profesionales del ámbito de la discapacidad intelectual, 2.000 personas con discapacidad intelectual y más de 400 familiares) y en general a una mayor concienciación social de esta problemática.

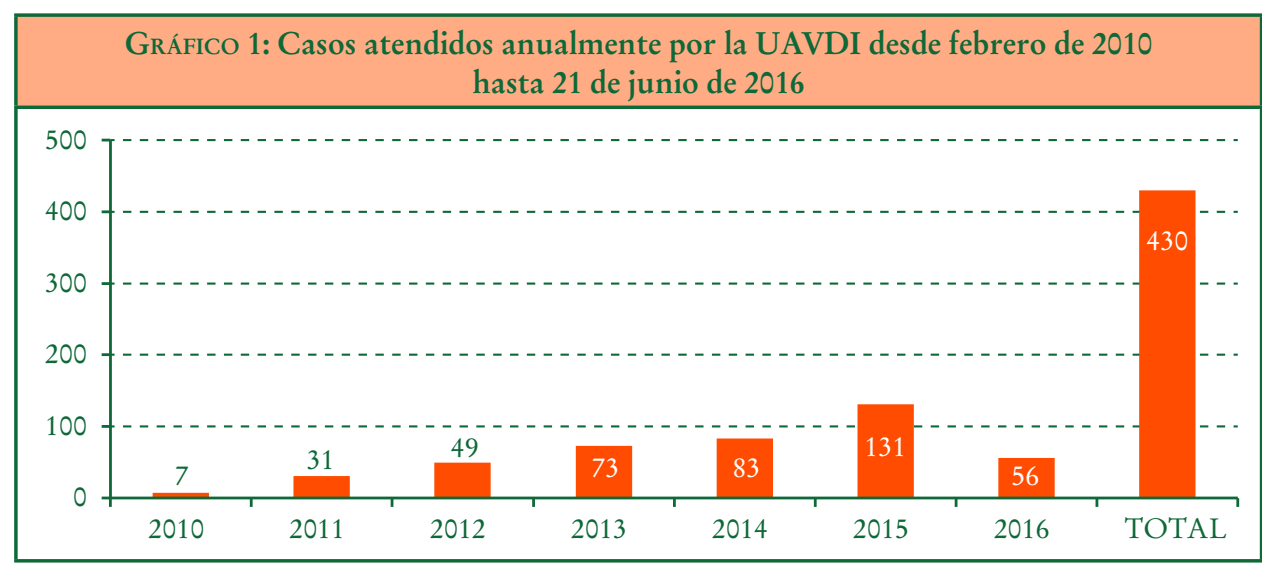

Respecto al perfil de las personas con discapacidad intelectual atendidas, el $72 \%$ de las víctimas que atiende la UAVDI son mujeres (ver Gráfico 2), lo que probablemente se deba a la doble vulnerabilidad a la que están sujetas, por un lado, por su condición de discapacidad y, por el otro, por su condición de mujer. Cabe señalar también en este punto las grandes dificultades con las que se encuentran los hombres para poder solicitar ayuda cuando están siendo víctimas de cualquier forma de abuso o maltrato, en especial el sexual, pues a los sentimientos propios de cualquier víctima se suma una terrible estigmatización.

Por otro lado, más de la mitad (63\%) de los casos recibidos por la unidad son adultos con discapacidad intelectual (ver Gráfico 2). La difícil labor de detectar un caso

GRÁFICO 2: Sexo y edades de las personas con discapacidad intelectual atendidas por la UAVDI

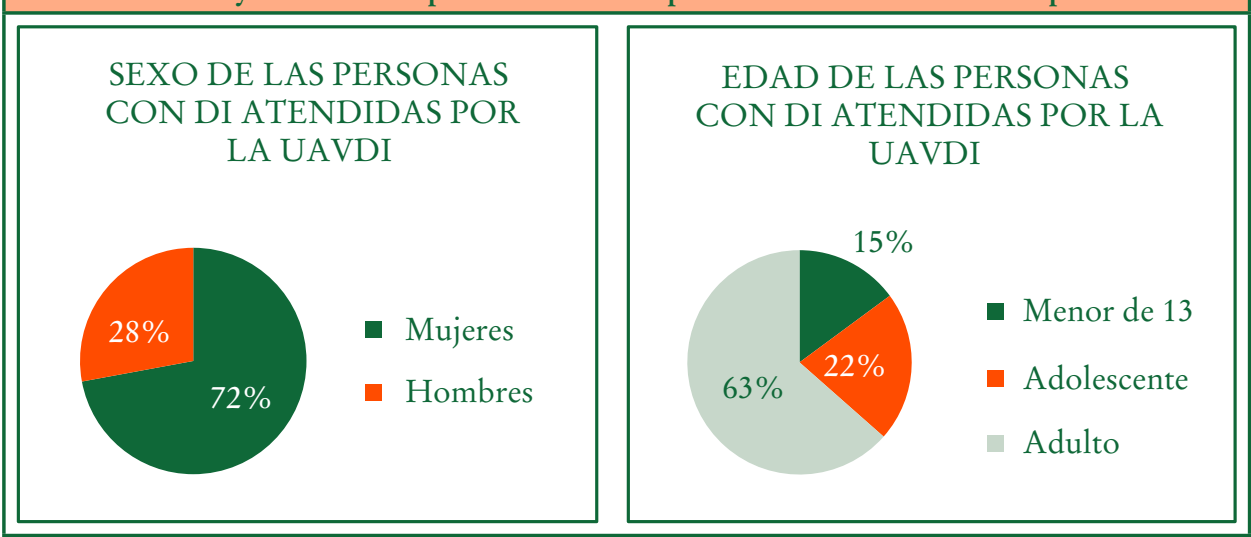

(C) Ediciones Universidad de Salamanca / CC BY-NC-ND

Siglo Cero, vol. 48 (1), n. ${ }^{\circ}$ 261, 2017, enero-marzo, pp. 37-49 
de abuso a una persona con discapacidad intelectual se hace todavía más compleja cuando ésta es menor de edad. Algunos de los factores que pueden explicar este hecho son las mayores relaciones de dependencia entre la víctima y el autor o la menor credibilidad que se le da a la palabra de un menor (Cederborg y Gumpert, 2010). Una vez más, en los casos de menores de edad hablaríamos de una doble vulnerabilidad, o incluso triple, si hacemos referencia a mujeres menores de edad.

\section{Vulnerabilidad de las personas con discapacidad intelectual frente al proceso policial y judicial}

Una de las labores más difíciles y complejas de los operadores jurídicos es garantizar que la obtención del testimonio de la víctima y los testigos sea lo más completa y precisa posible. Cuando se trata de obtener un testimonio de una persona con discapacidad intelectual, la tarea se vuelve realmente compleja (Cederborg y Lamb, 2008; Recio, Alemany y Manzanero, 2012). Recio et al. (2012) realizaron una revisión de los factores que hacen esta labor tan difícil: la menor cantidad de detalles que la persona con discapacidad intelectual, por regla general, incorpora en sus relatos (Bettenay, Ridley, Henry y Crane, 2014; Henry, Ridley, Perry y Crane, 2011; Henry y Gudjonsson, 2003; Perlman, Ericsson, Esses y Isaacs, 1994); las dificultades que afloran ante la formulación de preguntas cerradas (Bull, 1995; Milne y Bull, 2006; Perlman et al., 1994); o la tendencia a la aquiescencia (Clare y Gudjonsson, 1995). Pero el acceso a la justicia de las personas con discapacidad intelectual no sólo se ve afectado por la complejidad que supone la obtención de su testimonio. Las dilaciones excesivas de los procesos, en conjunción con la posible afectación de la memoria autobiográfica de la persona con discapacidad intelectual, pueden resultar dramáticas, así como la aplicación de técnicas de evaluación de la credibilidad -como la CBCA (Steller, 1989)-, que han mostrado no ser funcionales con personas con discapacidad intelectual (Recio et al., 2012) y, por tanto, también suponen una barrera para su acceso a la justicia. Estas dificultades, entre las que se encuentra la menor credibilidad, hacen de las personas con discapacidad intelectual unas víctimas especialmente vulnerables (Reiter et al., 2007).

Por otro lado, se debe considerar que el paso por el sistema de justicia supone un gran desgaste emocional para cualquier víctima, en ocasiones incluso más traumático que el producido por la propia situación de victimización criminal, provocando el llamado efecto de revictimización o victimización secundaria (Botero, Coronel y Pérez, 2009). En el caso de las personas con discapacidad intelectual, como señalan Botero et al. (2009), durante el interrogatorio y las diferentes pruebas indagatorias, el sistema judicial muchas veces no tiene en cuenta los aspectos cognitivos y afectivos de la víctima. Este hecho provoca que el efecto de la revictimización sea todavía más nocivo en las víctimas con discapacidad intelectual.

Parece claro que, en los casos en los que la víctima tiene discapacidad intelectual, se hace necesario el diseño de un procedimiento orientado a las necesidades de la víctima para reducir el efecto de la revictimización (Shapland, Willmore y Duff, 1985) y garantizar un acceso a la justicia en condiciones de igualdad (Recio et al., 2012). 
Por todo ello, y para garantizar que cuando una víctima con discapacidad intelectual pasa por el sistema de justicia la obtención de la prueba testifical se dé en las mejores condiciones y que las diferentes barreras a las que se enfrenta la víctima con discapacidad intelectual se vean superadas, así como que se reduzca al máximo el efecto de la revictimización, en 2010 la Fundación Carmen Pardo-Valcarce pone en marcha su servicio de facilitadores (Recio et al., 2012).

En lo que se refiere al acceso a la justicia de las personas con discapacidad intelectual, ya la Convención sobre los Derechos de las Personas con Discapacidad de Naciones Unidas (en adelante, CDPD) señala en su artículo 13 que "[...] las personas con discapacidad tengan acceso a la justicia en condiciones de igualdad, incluso mediante ajustes de procedimiento [...]”. Por otro lado, la entrada en vigor en 2015 de la Ley 4/2015, de 27 de abril, del Estatuto de la Víctima del Delito introduce específicamente muchas de las adaptaciones procedimentales que propone el facilitador (Recio et al., 2012). Algunas de las consideraciones que se recogen en el Estatuto de la Víctima del Delito y que velan por la adaptación del proceso a las necesidades de la persona con discapacidad intelectual son: el derecho a entender y ser entendido (art. 4), la necesidad de una evaluación individual de la condición de la persona (art. 23) o la declaración a través de profesionales con formación específica (art. 25.1).

\section{Análisis de los casos de abuso sexual}

De los 430 casos atendidos por la UAVDI hasta la fecha, el 71,16\% son relativos a un delito contra la libertad o indemnidad sexuales (ver Gráfico 3).

\begin{tabular}{|c|c|c|c|c|}
\hline \multicolumn{5}{|c|}{ GrÁfICo 3: Porcentaje de abuso sexual en las personas atendidas por la UAVDI } \\
\hline \multicolumn{5}{|c|}{ PORCENTAJE DE ABUSO SEXUAL EN LAS PERSONAS ATENDIDAS POR LA UAVDI } \\
\hline \multicolumn{2}{|c|}{ Tipo de abuso } & Casos & Porcentaje & \\
\hline \multicolumn{2}{|c|}{ ABUSO SEXUAL } & 306 & $71,16 \%$ & \multirow{10}{*}{$\begin{array}{l}\text { PORCENTAJE DE ABUSO SEXUAL EN } \\
\text { LAS PERSONAS ATENDIDAS POR LA } \\
\text { UAVDI }\end{array}$} \\
\hline \multicolumn{2}{|c|}{$\begin{array}{l}\text { OTROS ABUSOS (casos con } \\
\text { múltiples situaciones de abuso) }\end{array}$} & \multirow{9}{*}{124} & \multirow{9}{*}{$28,84 \%$} & \\
\hline TIPO DE ABUSO & CASOS & & & \\
\hline Trata de seres humanos & 39 & & & \\
\hline Violencia de género & 39 & & & \\
\hline Violencia doméstica & 40 & & & \\
\hline Maltrato físico & 30 & & & \\
\hline Maltrato psicológico & 25 & & & \\
\hline Abuso económico & 15 & & & \\
\hline Negligencia & 20 & & & \\
\hline \multicolumn{2}{|l|}{ TOTAL } & 430 & $100 \%$ & \\
\hline
\end{tabular}

De las 306 personas con discapacidad intelectual que referían haber sufrido un abuso sexual, el $74,84 \%$ han sido mujeres (ver Tabla 1 ). 
TABLA 1: Sexo y edades de las personas con discapacidad intelectual atendidas por la UAVDI por abuso sexual

\begin{tabular}{|c|c|c|c|c|c|}
\hline \multicolumn{3}{|c|}{$\begin{array}{l}\text { PERFIL EN FUNCIÓN DE LA EDAD } \\
\text { DE LAS PERSONAS CON DI ATENDIDAS } \\
\text { POR LA UAVDI POR ABUSO SEXUAL }\end{array}$} & \multicolumn{3}{|c|}{$\begin{array}{l}\text { PERFIL EN FUNCIÓN DEL SEXO } \\
\text { DE LAS PERSONAS CON DI ATENDIDAS } \\
\text { POR LA UAVDI POR ABUSO SEXUAL }\end{array}$} \\
\hline Tramo de Edad & $N$ & Porcentaje & Sexo & $N$ & Porcentaje \\
\hline Menor de 13 años & 58 & $18,95 \%$ & Mujeres & 229 & $74,84 \%$ \\
\hline Adolescente & 69 & $22,55 \%$ & Hombres & 77 & $25,16 \%$ \\
\hline Adulto & 179 & $58,50 \%$ & & & \\
\hline
\end{tabular}

En cuanto a la relación entre la persona con discapacidad intelectual que refiere haber sufrido el abuso sexual y el supuesto autor, destaca que el $41,83 \%$ de los casos pertenecen al ámbito familiar. Es decir, la relación entre la supuesta víctima y el presunto autor, en 128 casos, es de parentesco (padre, madre, hermano, hermana, abuelo...). Los otros tipos de vinculación son la extrafamiliar por parte de un desconocido, con un $27,12 \%$ de los casos; la extrafamiliar por parte de un igual (compañero del colegio, del trabajo, del Centro Ocupacional, del recurso residencial...), con un 20,59\% de los casos; y la extrafamiliar por parte de un profesional (profesor, psicólogo o educador), con un 10,46\% de los casos (ver Gráfico 4).

\begin{tabular}{|c|c|c|c|c|}
\hline \multicolumn{3}{|c|}{ GrÁFICO 4: Relación supuesto autor y supuesta víctima de abuso sexual de los casos } \\
atendidos por la UAVDI
\end{tabular}

() Ediciones Universidad de Salamanca / CC BY-NC-ND

Siglo Cero, vol. 48 (1), n. ${ }^{\circ}$ 261, 2017, enero-marzo, pp. 37-49 
5. Intervención del facilitador en casos de abuso sexual a personas con discapacidad intelectual

A continuación, se hace un análisis descriptivo de los principales datos que se desprenden del paso de las personas con discapacidad intelectual que refieren un abuso sexual por el sistema de justicia y de cómo influye la intervención del facilitador en la consecución del proceso judicial.

Cabe destacar que no todos los casos que son atendidos por la UAVDI son denunciados. Indicar que el Código Penal establece que determinados delitos, como algunos delitos contra libertad e indemnidad sexuales, son considerados semipúblicos, por lo que es un derecho y no un deber de la víctima -o su representante legal- el poder denunciarlos.

De esta manera, de los 306 casos atendidos por la UAVDI que refieren un abuso sexual, han sido denunciados menos de la mitad de los mismos, el 39,2\% (ver Gráfico 5), lo que supone que 120 personas con discapacidad intelectual -o sus representantes legales- dan el complejo paso de denunciar. Los datos que otros investigadores nos ofrecen en cuanto al porcentaje de casos de abuso sexual en personas sin discapacidad intelectual que son denunciados son los siguientes: Finkelhor (2009) indica que aproximadamente el 30\% de los casos de agresión sexual son denunciados y Martínez (2012) apunta que sólo el 15\% de los abusos sexuales a menores es denunciado.

Por tanto, volviendo al abuso sexual a personas con discapacidad intelectual, los datos obtenidos en los casos atendidos por la UAVDI y el porcentaje de denuncia se sugieren elevados. Este hecho puede apuntar a que la oportunidad que le ofrece la UAVDI a la persona con discapacidad intelectual de velar por la adaptación del proceso judicial a sus características, a través del facilitador, funciona como un efecto motivador de cara a la posibilidad de la denuncia o que los casos que llegan a la UAVDI

\begin{tabular}{|c|c|c||c|c||}
\hline \multicolumn{5}{|c|}{$\begin{array}{c}\text { GrÁfICO 5: Casos denunciados de abuso sexual en los que interviene un facilitador } \\
\text { de la UAVDI }\end{array}$} \\
\hline FACILITADOR & $\mathrm{N}$ & $\%$ & $\begin{array}{c}\text { Porcentaje de intervención del } \\
\text { facilitador en casos de abuso } \\
\text { sexual atendidos por la UAVDI }\end{array}$ \\
\hline INTERVIENE & 60 & $50 \%$ & \\
\hline NO INTERVIENE & 60 & $50 \%$ & \\
\hline TOTAL & 120 & $100 \%$ & \\
\hline
\end{tabular}

(C) Ediciones Universidad de Salamanca / CC BY-NC-ND

Siglo Cero, vol. 48 (1), n. ${ }^{\circ} 261,2017$, enero-marzo, pp. 37-49 
son aquellos que ya tienen una predisposición al acompañamiento, quedando otros enterrados en este problema social que aún no acaba de aflorar del todo.

El proceso de inserción de apoyos por parte del facilitador no siempre es fácil pues la posibilidad de la incorporación del facilitador al proceso no está garantizada. Si bien, como se ha mencionado anteriormente, la CDPD de Naciones Unidas y nuestro Estatuto de la Víctima del Delito contemplan la posibilidad de insertar en el proceso judicial la figura de "un profesional experto", los operadores jurídicos no siempre consideran esta posibilidad y no siempre posibilitan que el facilitador diseñe e implemente los “ajustes de procedimiento necesarios” para garantizar un acceso a la justicia en condiciones de igualdad de la persona con discapacidad intelectual.

De hecho, desde la puesta en marcha de la UAVDI, tan solo en el 50\% (ver Gráfico 5) de los casos denunciados por abuso sexual a una persona con discapacidad intelectual ha sido posible introducir la figura del facilitador. Su intervención, en estos 60 casos, aunque no siempre ha podido abarcar la totalidad del proceso judicial, ha permitido que se inserten determinados "ajustes de procedimiento", tales como la prueba preconstituida o la obtención del testimonio a través de su figura.

Pero, ¿qué ocurre cuando el facilitador inserta sus apoyos en un proceso judicial en el que una persona con discapacidad intelectual refiere un abuso sexual? En primer lugar, los efectos reductores del impacto de la victimización secundaria son claros (Recio et al., 2012).

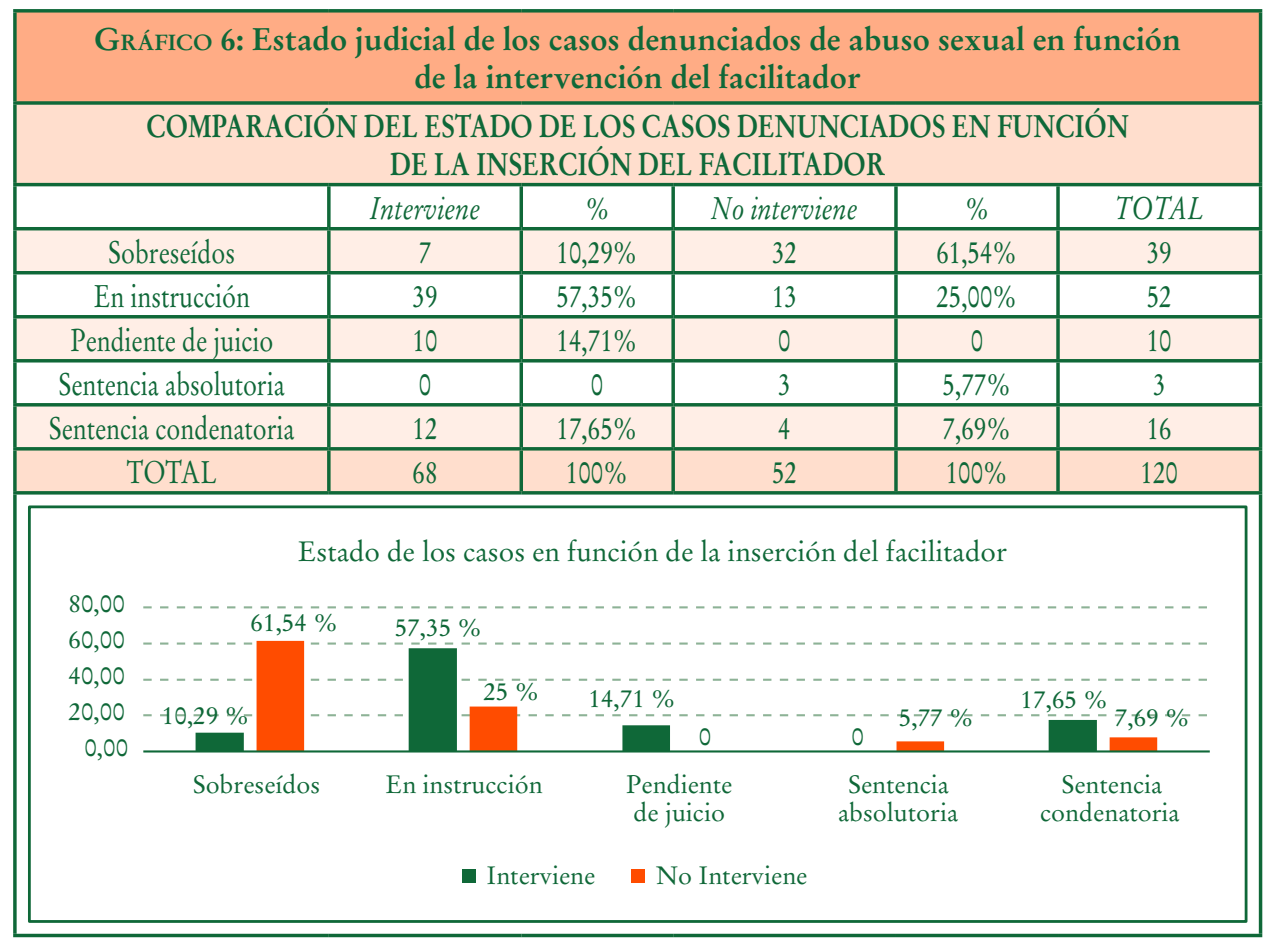


Y, sobre todo, podemos concluir que se mejora significativamente el acceso a la justicia. Mientras más del $60 \%$ de los casos de abuso sexual en los que el facilitador no ha tenido oportunidad de insertar sus apoyos han sido sobreseídos en fase de instrucción(ver Gráfico 6), sólo el 10\% son sobreseídos cuando éste interviene. Las causas de los sobreseimientos suelen deberse a que, o bien el operador jurídico ha considerado que la información con la que cuenta es inconsistente y, por tanto, decide no proseguir con el proceso judicial, o bien no cuenta con la información necesaria para poder valorar la existencia de indicios de delito y, por tanto, no puede continuar con la persecución del supuesto delito. Parece claro que el tipo de entrevista se muestra crucial para mejorar la calidad del testimonio de una persona con discapacidad intelectual (Gentle, Milne, Powell y Sharman, 2013).

Otro factor que se muestra relevante en ese $60 \%$ es el tipo de relación existente entre el supuesto autor y la persona con discapacidad intelectual que revela un abuso sexual. El impacto del facilitador sobre este factor se muestra crucial. Del total de casos atendidos por la UAVDI de abuso sexual intrafamiliar que han sido sobreseídos, en el 93,75\% de ellos no ha intervenido el facilitador, en comparación con el 6,25\% de casos sobreseídos cuando ha intervenido el facilitador (ver Gráfico 7).

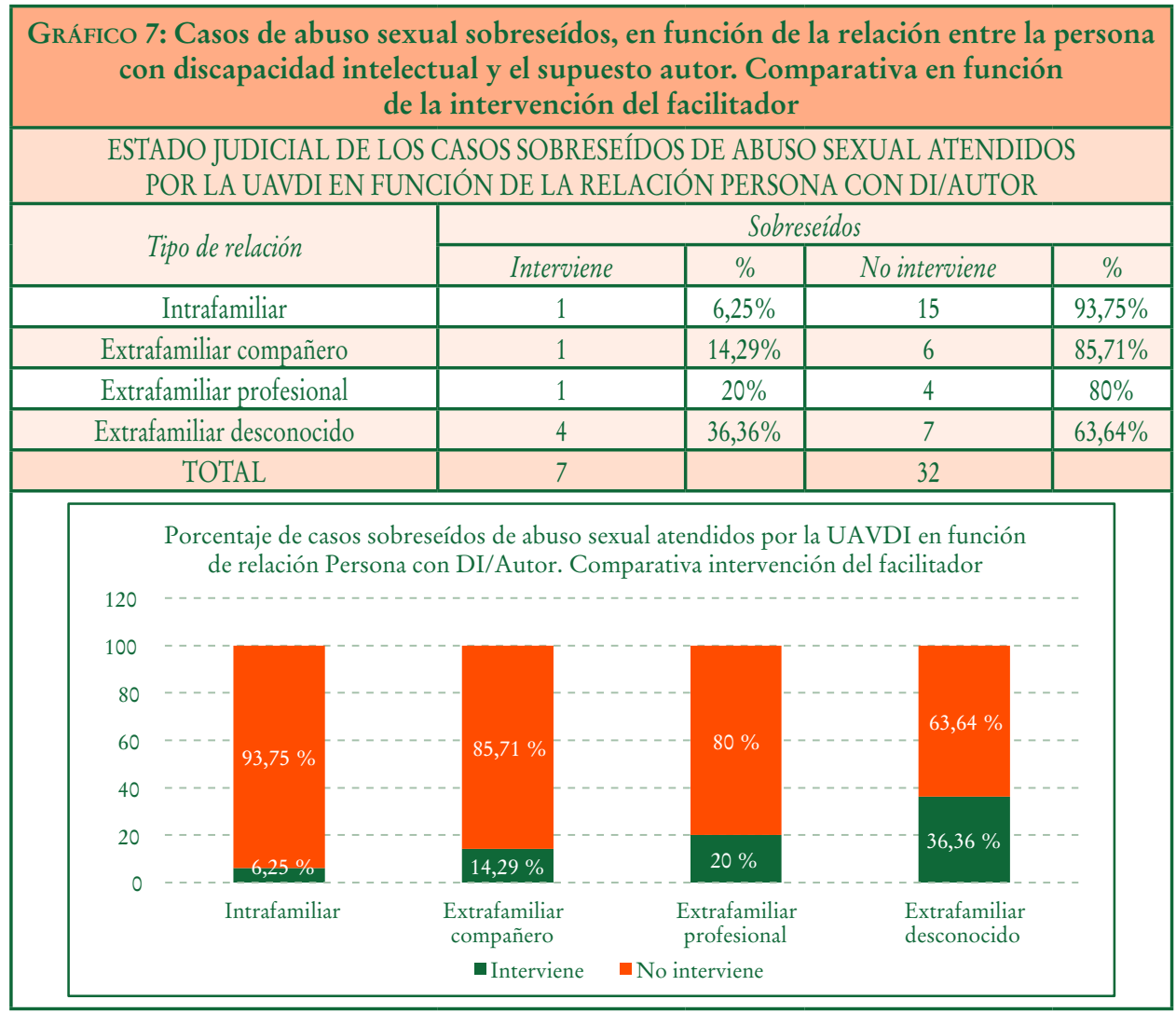

(C) Ediciones Universidad de Salamanca / CC BY-NC-ND

Siglo Cero, vol. 48 (1), n. ${ }^{\circ}$ 261, 2017, enero-marzo, pp. 37-49

$$
-46-
$$


Pero, ¿por qué se muestra tan determinante la actuación del facilitador en los casos de abuso sexual intrafamiliar? Cuando se trata de un abuso sexual intrafamiliar, a las dificultades que entraña el proceso judicial para la persona con discapacidad intelectual se le debe sumar el conflicto que, en muchas ocasiones, implica el tener un vínculo estrecho con el supuesto agresor (padre/hijo, hermano/hermana, abuelo/nieta...), y que por tanto debe también abordarse en el proceso. En estos casos, la intervención del facilitador se muestra crítica, pues también acompaña en la elaboración y abordaje de la culpa derivada de las posibles consecuencias que el proceso puede tener sobre familiares cercanos.

\section{Conclusiones}

En la actualidad las personas con discapacidad intelectual, desafortunadamente, siguen encontrando demasiadas barreras en la totalidad de las esferas de la sociedad. El sistema de justicia no es precisamente una excepción y los obstáculos que tienen que superar cuando acceden al sistema de justicia son innumerables. Si queremos garantizar que las personas con discapacidad intelectual sean ciudadanos de pleno derecho y que nuestro sistema judicial respete de manera efectiva el Principio de Igualdad, se hace necesaria la inclusión de todos los “ajustes de procedimiento necesarios" que posibiliten que el acceso a la justicia de una persona con discapacidad intelectual no se convierta en proceso traumático ni en algo inalcanzable.

La Ley 4/2015, de 27 de abril, del Estatuto de la Víctima del Delito ha supuesto un hito en el ordenamiento jurídico español en lo que se refiere a los derechos de las víctimas. Afortunadamente, el Estatuto recoge en muchos de sus artículos la posibilidad de insertar apoyos, en forma de adaptaciones en el proceso, que posibiliten que las personas con discapacidad intelectual puedan superar las innumerables barreras que tienen que hacer frente cuando, después de haber padecido una vulneración de sus derechos en forma de abuso o maltrato, deciden denunciar.

La figura del facilitador se ha mostrado como un garante en la inserción de estos apoyos. Por tanto, la inclusión de este profesional en los procesos en los que la víctima tenga discapacidad intelectual supondrá un hito fundamental para que España cumpla con la Convención sobre los Derechos de las Personas con Discapacidad en materia de accesibilidad a la justicia y será un paso adelante en el largo camino que queda por recorrer para conseguir que las personas con discapacidad intelectual sean ciudadanos de pleno derecho.

\section{Referencias bibliográficas}

Anaut-Bravo, S., Arza, J. y Álvarez, M. J. (2014). La incidencia de la exclusión social en los hogares con personas con discapacidad. En M. LAPARRA, VII Informe sobre exclusión y desarrollo social en España, documento de trabajo 3.3.

Bettenay, C., Ridley, A. M., Henry, L. A. y Crane, L. (2014). Cross-examination: The testimony of children with and without Intellectual Disabilities. Applied Cognitive Psychology, $28,204-214$. 


\section{LA FIGURA DEL FACILITADOR EN CASOS DE ABUSO SEXUAL A PERSONAS CON DISCAPACIDAD INTELECTUAL \\ ALMUDENA MARTORELL CAFRANGA Y ALBERTO ALEMANY CARRASCO}

Botero, C., Coronel, E. y Andrés, C. (2009). Revisión teórica del concepto de victimización secundaria. Liberabit, Revista de Psicología, 8 (1), 49-58.

Brown, H., Stein, J. y Turk, V. (1995). The sexual abuse of adults with learning disabilities. Mental Handicap Research, 8, 3-24.

Bull, R. (2010). The investigative interviewing of children and other vulnerable witnesses: Psychological research and working/profesional practice. Legal and Criminological Psychology, 15, 5-23.

Cederborg, A. C. y Gumpert, C. (2010). The challenge of assessing credibility when children with intellectual disabilities are alleged victims of abuse. Scandinavian Journal of Disability Research, 12 (2), 125-140.

Cederborg, A. C. y LAmb, M. (2008). Interviewing alleged victims with intellectual disabilities. Journal of Intellectual Disability Research, 52 (1), 49-58.

Clare, I. C. H. y Gudjonsson, G. H. (1995). The vulnerability of suspects with intellectual disabilities during police interviews: A review and experimental study of decision-making. Mental Handicap Research, 8, 110-128.

Finkelhor, D. (2009). The Prevention of Childhood Sexual Abuse. Future of Children, 19 (2), 169-94.

Gentle, M., Milne, R., Powell, M. B. y Sharman, S. J. (2013). Does the cognitive Interview Promote the Coherence of Narrative Accounts in Children with and Without an Intellectual Disability? International Journal of Disability, Development and Education, 60, 30-34.

Henry, L. A. y Gudjonsson, G. H. (2003). Eyewitness memory, suggestibility and repeated recall sessions in children with mild and moderate intellectual disabilities. Law and Human Behavior, 27, 481-505.

Henry, L., Ridley, A., Perry, J. y Crane, L. (2011). Perceived credibility and eyewitness testimony of children with intellectual disabilities. Journal of Intellectual Disability Research, 55 (4), 385-391.

Horner-Johnson, W. y Drum, C. E. (2006). Prevalence of maltreatment of people with intellectual disabilities: a review of recent published research. Mental Retardation and Developmental Disabilities Research Review, 12, 57-69.

Huete, A. (2012). La discapacidad como factor de exclusión social. Evidencias empíricas desde una perspectiva de derechos. Salamanca: Universidad de Salamanca.

Johnson, H. (2012). Protecting the most vulnerable from abuse. The ASHA Leader, 17 (14), 16-19.

Martínez, M. I. (2012). Abuso sexual en niños y adolescentes. Palma de Mallorca: Criminología y Justicia.

McCarthy, M. y Thompson, D. (1997). A Prevalence Study os Sexual Abuse of Adults with Intellectual Disabilities Referred for Sex Education. Journal of Applied Research in Intellectual Disabilities, 10 (2), 105-124.

Milne, R. y Bull, R. (2006). Interviewing victims of crime, including children and people with intellectual disabilities. En M. Kebbell y G. Davies (Eds.), Practical Psychology for forensic investigations (pp. 7-24). Chichester, WS: Wiley.

Perlman, N. B., Ericson, K. I., Esses, V. M. e Isaacs, B. J. (1994). The developmentally handicapped witness: Competecy as a function os question format. Law and Human Behaviour, $18,171-187$.

Recio, M., Alemany, A. y Manzanero, A. L. (2012). La figura del facilitador en la investigación policial y judicial con víctimas con discapacidad intelectual. Siglo Cero, 43 (3), 54-68.

(C) Ediciones Universidad de Salamanca / CC BY-NC-ND

Siglo Cero, vol. 48 (1), n. ${ }^{\circ}$ 261, 2017, enero-marzo, pp. 37-49 


\section{LA FIGURA DEL FACILITADOR EN CASOS DE ABUSO SEXUAL A PERSONAS CON DISCAPACIDAD INTELECTUAL \\ ALMUDENA MARTORELL CAFRANGA Y ALBERTO ALEMANY CARRASCO}

Reiter, S., Bryen, D. N. y Shachar, I. (2007). Adolescents with intelectual disabilities as victims of abuse. Journal of Intellectual Disabilities, 11, 371-87.

Shapland, J., Wilmore, J. y DufF, P. (1985). Victims andthe criminal justice system. Aldershot: Gower.

Sobsey, D. (1994). Violence and abuse in the lives of people with disabilities: The end of silent acceptance? Baltimore: Brookes.

Steller, M. (1989). Recent developments in statement analysis. En J. C. Yuille (Ed.), Credibility assessment (pp. 135-154). Netherland: Kluwer Academic Publishers.

Verdugo, M. Á., Alcedo, M. A., Bermejo, B. y Aguado, A. (2002). El abuso sexual a personas con discapacidad intelectual. Psicothema, 14, 124-129.

Verdugo, M. Á. y Bermejo, B. G. (1995). El maltrato en personas con retraso mental. En Personas con discapacidad. Perspectivas psicopedagógicas y rehabilitadoras (pp. 873-924). Madrid: Siglo Veintiuno. 